\title{
PARAGANGLIOMA PRÉ-AÓRTICO GIGANTE
}

\author{
Giant preaortic paraganglioma \\ Sergio Renato Pais COSTA, Rodrigo Nascimento PINHEIRO, Luciana Paganini PIAZZOLA, \\ Gustavo Henrique Soares TAKANO, Renato Arioni LUPINACCI
}

ABCDDV/627

Costa SRP, Pinheiro RN, Piazzola LP, Takano GHS, Lupinacci RA. Paraganglioma pré-aórtico gigante. ABCD Arq Bras Cir Dig 2008;21(4): 211-4 RESUMO - Introdução - O paraganglioma é um tumor neuroendócrino raro que ocorre mais frequentemente em adultos jovens. Geralmente produz catecolaminas acarretando síndrome adrenérgica. No entanto, muito raramente, quando não-funcionante seus sintomas são mais frequentemente associados à massa abdominal ou mesmo dor. Nessas circunstâncias costumam representar diagnóstico difícil sendo confundidos com os sarcomas de retroperitônio. Relato do caso - Paraganglioma pré-aórtico gigante não-funcionante em paciente com níveis baixos de catecolaminas (sérico e urinário). O diagnóstico pré-operatório foi dado por tomografia computadorizada, onde foi observada massa sólida, hipervascular com calcificações e área cística central. O paciente foi submetido à ressecção cirúrgica da massa com boa evolução pós-operatória. Um ano após a operação, o doente encontra-se vivo sem recidiva tumoral. Conclusão - Em que pese a raridade o paraganglioma pré-aortico não-funcionante deve ser lembrado com diagnóstico diferencial com os sarcomas de retroperitônio. Seu tratamento é cirúrgico e apresenta bom prognóstico.

DESCRITORES - Paraganglioma. Diagnóstico por imagem.

\section{INTRODUÇÃO}

O paraganglioma também denominado quimiodectoma é neoplasia relativamente rara, geralmente benigna que se origina no tecido quimiorreceptor parassimpático do corpo carotídeo, glomo jugular, glomo timpânico e corpos aórticos. É constituída histologicamente por células hipercromáticas arredondadas ou ovóides que tendem a agrupar-se em padrão alveolar dentro de uma quantidade escassa a moderada de estroma fibroso e alguns canais vasculares de paredes delgadas. Estes tumores podem produzir catecolaminas e frequentemente estão associados à síndrome adrenérgica. Quando isto ocorre são consequentemente denominados de tumores funcionais. Contudo, em raras situações, não produzem catecolaminas ou seus metabólitos determinando sintomatologia mais frequentemente associada ao efeito de massa tumoral. Dor ou tumoração palpável são os sintomas mais comuns nessas circunstâncias. A incidência de malignidade nesses tumores é baixa, em torno de 10-15\%. A diferenciação histológica entre os paragangliomas benignos e malignos costuma ser problemática, haja vista que determinados marcadores histológicos como pleomorfismo celular e figuras mitóticas entre outros costumam ser vistos em ambas as situações clínicas. Assim sendo como ocorre também com demais tumores neuroendócrinos, a definição de malignidade está mais associada ao seu comportamento biológico do

Trabalho realizado no Hospital Servidor Público Estadual de São Paulo, São Paulo, SP, Brasil.

Correspondência: Dr. Sergio Renato Pais Costa, e-mail sergiorenatopais@ig.com.br que propriamente aos achados histológicos. A invasão de estruturas contíguas e metástases à distância determinam a malignidade de sua origem. Nos tumores funcionantes, seu diagnóstico pode ser dado pela elevação das catecolaminas (urinárias ou séricas) bem como de seus metabólitos.

Em contrapartida, quando não-funcionantes, o diagnóstico diferencial com os sarcomas de retroperitônio costuma ser difícil e os exames de imagem como tomografia computadorizada (TC) e ressonância nuclear magnética (RNM) podem contribuir o seu diagnóstico $3,6,7,10$.

\section{RELATO DO CASO}

Paciente de 38 anos de idade, do sexo masculino e caucasiano, deu entrada com queixa de massa abdominal palpável há três meses. Era portador de hipertensão arterial sistêmica compensada (HAS). Ao exame físico, a massa de aproximadamente 15 por $13 \mathrm{~cm}$ em seus maiores eixos de limites mal definidos e fibroelástica era localizada em hipocôndrio esquerdo e se prolongava em direção à região do epigástrio. Inicialmente foram realizadas para investigação desse quadro clínico uma endoscopia digestiva alta e ultra-sonografia de abdome total que não evidenciaram alterações. Subsequentemente foi realizada uma tomografia computadorizada de abdome que mostrou massa regular sólida hipervascular, com área de necrose central com calcificações e contorno periférico bem definido com realce homogêneo a administração do contraste endovenoso. A referida lesão era localizada no retroperitônio em topografia de rim esquerdo - adrenal esquerda (sem definição precisa de sua origem), contudo apresentava-se ântero-lateralmente 
à aorta abdominal e veia cava inferior se estendendo desde $\mathrm{o}$ tronco celíaco até a bifurcação da aorta (Figura 1).

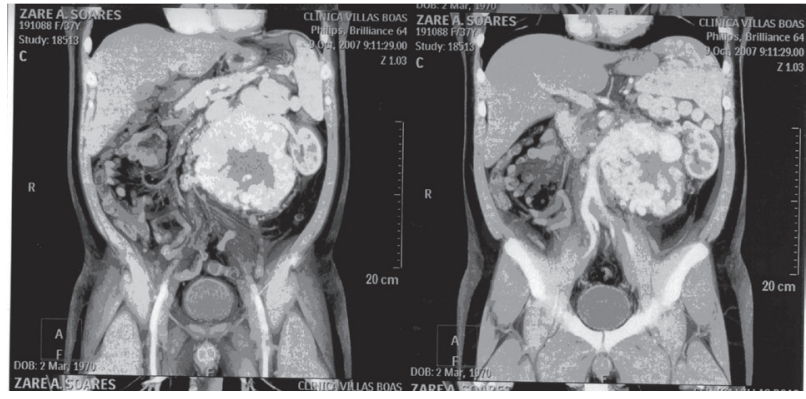

FIGURA 1 - TC de abdome com contraste - Grande massa tumoral calcificada com bordas bem delimitadas junto à aorta abdominal apresentado realce em sua periferia após a administração do contraste endovenoso, além da grande área cística central (necrose)

Foram dosados os marcadores tumorais séricos antígeno carcinoembrionário (CEA) e CA 19, 9 bem como catecolaminas e metanefrinas. Também foram dosados na urina de 24 horas: ácido vanilmandélico, metanefrinas e catecolaminas livres. Foram realizadas três medidas consecutivas em intervalos de uma semana em busca de neoplasia neuro-endócrina funcionante. Em todas as medidas não foi observado alterações significantes das respectivas dosagens. Sendo assim baseado nas suas características radiológicas (massa hipervascular, calcificada e com necrose central), a principal hipótese diagnóstica pré-operatória era de tumor neuroendócrino não-funcionante (paraganglioma) e como diagnóstico diferencial um sarcoma de retroperitônio. Como se tratava de tumor não-secretor não foi necessário preparo pré-operatório específico no que tange a bloqueio alfa ou beta-adrenérgico. Consequentemente, o paciente foi submetido à laparotomia exploradora para ressecção da massa. Ao inventário intra-operatório foi observada uma lesão de 15 X $15 \mathrm{~cm}$ de diâmetro, de natureza predominantemente sólida, altamente vascularizada, com áreas císticas e hemorrágicas de permeio que parecia envolver tanto o rim esquerdo quanto a adrenal esquerda. Como havia dúvida no sítio de origem do tumor e em sua margem, optou-se pela ressecção em monobloco dessas estruturas aparentemente envolvidas. Por conseguinte foi realizada ressecção em monobloco do tumor com nefrectomia radical esquerda e linfadenectomia para-aórtica. O paciente evoluiu sem complicações e teve alta no sétimo dia pós-operatório.

Ao exame histológico foi evidenciada a presença de um tumor de $15 \times 15 \times 9 \mathrm{~cm}$ nos maiores eixos e de cerca de 2650 gr, com focos de hemorragia e necrose com intensa reação desmoplástica inflamatória e sua origem era neuroendócrina.

O achado histológico era compatível com paraganglioma pré-aórtico. As margens cirúrgicas eram livres de comprometimento neoplásico e não havia invasão da cápsula tumoral ou ainda embolização linfática ou vascular. Adicionalmente não foi evidenciada invasão do rim esquerdo ou da glândula supra-renal esquerda (apenas intensa atividade desmoplásticainflamatória) e tão pouco comprometimento de linfonodos. Assim sendo esse tumor foi classificado como benigno.
O painel imunoistoquímico confirmou a origem neuroendócrina do tumor (Tabela 1 e Figuras 2,3). Por conseguinte, o paciente foi submetido a mapeamento de corpo total com MIBG- I 131 e não se observou neoplasia captante residual local ou à distância. Consequentemente não foi realizado qualquer tratamento adjuvante. O doente foi seguido com exames de imagem periodicamente (cada três meses) e não se observou recidiva da doença local ou sistêmica até um ano após sua ressecção. Encontra-se assintomático em relação à massa abdominal e segue controlado da hipertensão arterial com uso de medicação anti-hipertensiva.

TABELA 1 - Painel imunoistoquímico

\begin{tabular}{ll}
\hline Marcador Imunoistoquímico & \\
\hline Cromogranina A & Positivo \\
HMB-45 & Negativo \\
AE1/AE3 (Citoqueratinas) & Negativo \\
Desmina & Negativo \\
Sinaptofisina & Positivo \\
S100 & Positivo (nas células sustentaculares) \\
Antígeno HepPar-1 & Negativo \\
\hline
\end{tabular}

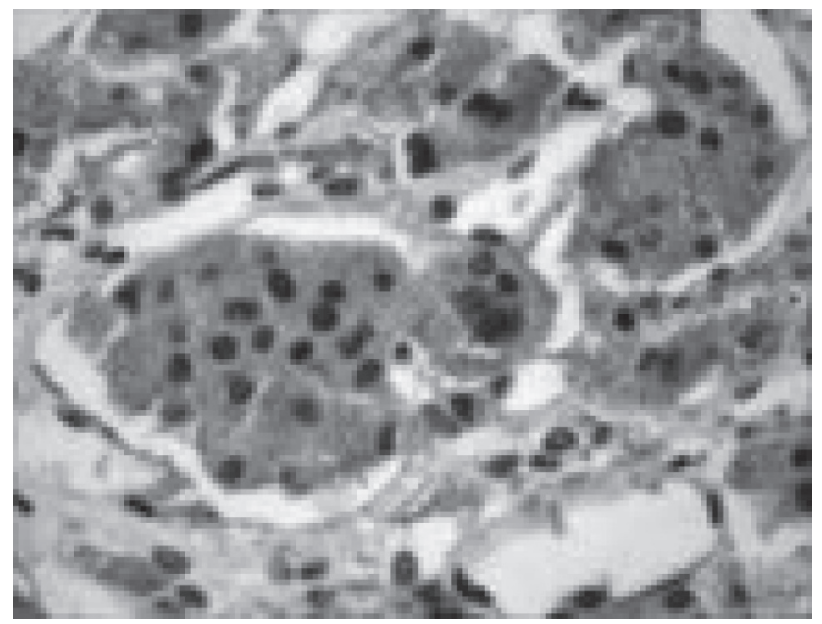

FIGURA 2 - Reação positiva para Cromogranina - 40X

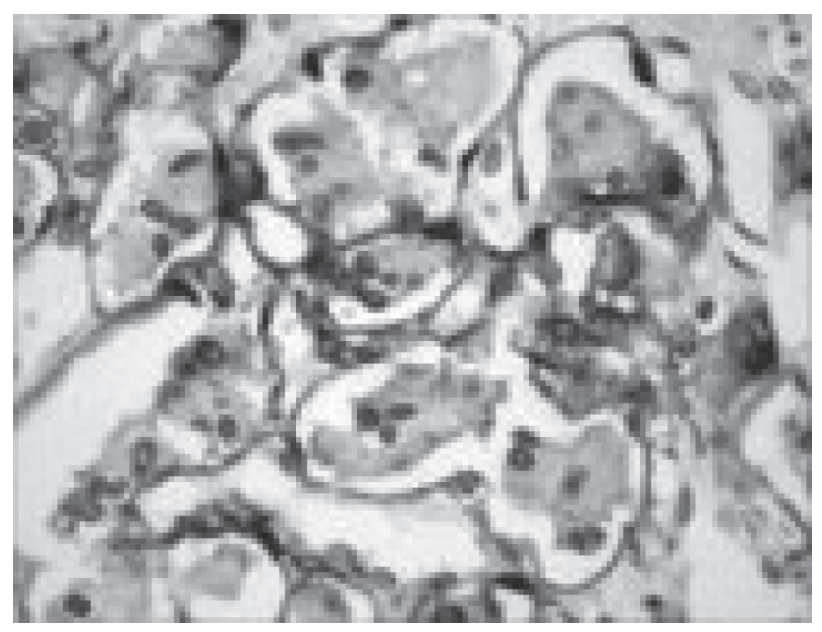

FIGURA 3 - Reação positiva para Proteína S-100 - 40 X 


\section{DISCUSSÃO}

Os paragangliomas retroperitoniais podem ter origem na glândula supra-renal ou nos gânglios simpáticos aórticos (extra-supra-renal). Estes tumores podem estar associados a diversas síndromes como NEM tipo IIA, Von Hippel-Lindau, neurofibromatose. Também tem sido associado a outros tumores, como carcinóides gastrointestinal ou brônquico ${ }^{5,10}$.

Conforme observado no presente caso e de acordo com Pui et al. ${ }^{8}$, este tumor apresenta preponderância no sexo masculino sendo mais frequentemente observado em adultos jovens (menor que 40 anos). Dentre os paragangliomas retroperitoniais, os de origem extra-supra-renal são os mais raros (29\%) sendo que destes somente $24 \%$ apresentam sinais claros de malignidade. Ao contrário do observado, quando malignos são mais frequentemente não-funcionais e apresentam níveis normais de ácido vanil-mandélico urinário ${ }^{9,10}$.

A maioria dos paragangliomas retroperitoniais é funcional, particularmente nos tumores de localização adrenal que são em sua maioria benignos (81\%). As catecolaminas plasmáticas, as metanefrinas urinárias e também o ácido vanil-mandélico urinário costumam estar elevados nesses tumores. Segundo Piu et al. 8, o tamanho dos tumores benignos ou funcionais tem sido significantemente menor $(6.4 \mathrm{~cm}$ e $6.1 \mathrm{~cm}$ respectivamente) do que o dos tumores malignos ou não-funcionais $(8.7 \mathrm{~cm}$ e $7.9 \mathrm{~cm}$ respectivamente). Da mesma forma, os tumores extra-adrenais também têm sido mais associados com malignidade (50\% X 13\%).

Em que pese o tamanho $(15 \mathrm{~cm})$ e a localização (extrasupra-renal) no presente caso, o tumor em questão apresentava características benignas como ausência de metástases ou invasão de estruturas contíguas $3,6,7,10$.

Aos exames de imagem, mormente a TC, os tumores benignos costumam ser mais homogêneos (46\%) e com margens mais bem definidas (92\%) como foi observado no presente caso. Já os tumores malignos são menos frequentemente homogêneos (25\%) e com margens mais bem definidas $(13 \%)^{3}$.

Assim como no presente caso, também tem sido observada em tumores volumosos, uma volumosa área de necrose central. Em que pese à raridade das calcificações que estão presentes em apenas $15 \%$ dos casos dos paragangliomas retroperitoniais, tal achado radiológico pode ajudar sobremaneira na elucidação diagnóstica como observado no presente $\operatorname{caso}^{2,6,10}$.

De maneira geral, os tumores neuroendócrinos são tumores bem vascularizados que captam avidamente o contraste na fase arterial tanto na TC quanto na RNM podendo também inclusive apresentar áreas císticas de permeio devido à necrose tumoral. Contudo, esses achados podem também ser observados em outros tumores retroperitoniais de origem neural ou mesodérmica, linfomas e metástases. Quando o paraganglioma é funcional, o diagnóstico pode ser mais facilmente realizado devido à elevação das catecolaminas e seus metabólitos em dosagens séricas ou urinárias ${ }^{4,8}$.

O ácido vanil-mandélico urinário e a dosagem sérica de catecolaminas têm apresentado altos índices de sensibilidade nessa situação $(95 \% \text { e } 100 \% \text {, respectivamente })^{9}$.

Nos tumores não-funcionais como no presente caso, a biópsia é necessária para o diagnóstico.

Embora possa ser realizado por punção, como seu tratamento é amiúde cirúrgico, a ressecção do tumor conjuntamente com estruturas envolvidas e linfonodos regionais pode ser realizado para os casos com imagem radiológica suspeita. Em situações como no presente caso em que haja dúvida em sua origem ou mesmo na suas margens de ressecção, a retirada em monobloco da lesão com estruturas contíguas é recomendável.

O mapeamento com metaiodobenzilguanidina (MIB I-131) pode auxiliar no diagnóstico tanto no tumor primário, quanto na lesão residual ou recidivada.

Essa neoplasia apresenta bom prognóstico e não há indicação de quimioterapia ou radioterapia adjuvante quando sua ressecção é completa. Esta prática deve ser reservada somente para os casos de paragangliomas malignos que se apresentem irressecáveis ou cujos doentes sejam inoperáveis ${ }^{3}$.

\section{CONCLUSÃO}

Embora raro, o paraganglioma pré-aórtico deve ser lembrado nas lesões expansivas oriundas do retroperitônio. A TC é útil para delinear a localização, extensão e natureza de determinadas lesões retroperitoniais. O diagnóstico diferencial com os sarcomas do retroperitônio deve ser lembrado. Seu tratamento é amiúde a ressecção cirúrgica total da lesão e apresenta bom prognóstico. 
Costa SRP, Pinheiro RN, Piazzola LP, Takano GHS, Lupinacci RA. Giant preaortic paraganglioma. ABCD Arq Bras Cir Dig 2008;21(4): 211-4

ABSTRACT - Racional - Paraganglioma is a rare neuroendocrine tumor which often diagnosed in the young adult. Generally, paraganglioma produces catecholamines causing adrenergic syndrome. However, more rarely when tumor is nonfunctioning, their symptoms are more associated with an abdominal mass or even pain. In these circumstances, paraganglioma present a difficult diagnosis confounding with retroperitoneal sarcomas. Case report - The authors present a case of nonfunctioning giant preaortic paraganglioma. This patient had low levels of catecholamines (both seric and urinary). The preoperative diagnosis was done by means computed tomography. This lesion presented as well-vascularized tumor with calcifications and necrotic central area. The patient underwent a surgical resection with good postoperative outcome. To date, one year after surgical treatment, the patient is alive without recurrence. Conclusion - Despite its rarity, non-funcionating preaortic paraganglioma should be reminded with differential diagnosis with retroperitoneal sarcomas. Treatment is surgical resection and presents a good prognosis.

HEADINGS - Paraganglioma. Diagnostic imaging.

\section{REFERÊNCIAS}

1. Aguilo F, Tamayo N, Vazquez-Quintana E. Pheochromocytoma: A twenty-year experience at the University Hospital.PR Health Sci J 1991; 10: 135-42.

2. Asbury WL Jr, Hatcher PA, Gould HR, Reeves WA, Wilson DD. Bladder pheochromocytoma with ring calcification. Abdom Imaging 1996; 21: 275-7.

3. Gonzalez RJ, Lee JE. Adrenal Tumors. In: Feig BW, Berger DH, Fuhrman GM. The M.D. Anderson Surgical Oncology Handbook. Fourth Edition, Philadelphia: Lippincott \& Wilkins; 2006, p. 419-39.

4. Hayes WS, Davidson AJ, Grimley PM, Hartman DS. Extraadrenal retroperitoneal paraganglioma: Clinical, pathologic, and CT findings. AJR 1990; 155: $1247-50$.

5. Kryger-Baggesen N, Kjaergaard J, Sehested M. Nonchromaffin paraganglioma of the retroperitoneum. J Urol 1985; 134: 536-8.

6. Lane RH, Stephens DH, Reiman HM. Primary retroperitoneal neoplasms: CT findings in 90 cases with clinical and pathologic correlation. AJR 1989; 152: 83-9.
7. Patel YD, Morehouse HT. Malignant paragangliomas of the retroperitoneum: Value of computed tomography and angiography. Clin Radiol 1984; 35: 185-8.

8. Pui MH, Liu MJ, Guo Y, Chen YM. Computed Tomography of retroperitoneal paragangliomas. Australasian Radiology 1999; 43: 303-6.

9. Stephens DH, Sheedy IIPF, Hattery RR, Williamson B Jr. Diagnosis and evaluation of retroperitoneal tumors by computed tomography. AJR 1977; 129: 395-402.

10. Welch TJ, Sheedy IIPF, van Heerden JA, Sheps SG, Hattery RR, Stephens DH. Pheochromocytoma: Value of computed tomography.Radiology 1983; 148: $501-3$.

Fonte de financiamento: não há Conflito de interesse: não há Recebido para publicação: 21/05/2008 Aceito para publicação: 19/07/2008 